\title{
Non-Hermitian many-body topological excitations in interacting quantum dots
}

\author{
Timo Hyart $\odot^{1,2}$ and J. L. Lado $\odot^{1}$ \\ ${ }^{1}$ Department of Applied Physics, Aalto University, 00076 Aalto, Espoo, Finland \\ ${ }^{2}$ International Research Centre MagTop, Institute of Physics, Polish Academy of Sciences, Al. Lotników 32/46, 02-668 Warsaw, Poland
}

(Received 12 July 2021; accepted 11 January 2022; published 28 January 2022)

\begin{abstract}
Quantum dots are one of the paradigmatic solid-state systems for quantum engineering, providing an outstanding tunability to explore fundamental quantum phenomena. Here we show that non-Hermitian many-body topological modes can be realized in a quantum dot chain by utilizing a gate-tunable modulation of dissipation, and they emerge purely because of the non-Hermiticity. By exactly solving the non-Hermitian interacting description both with exact diagonalization and tensor networks, we demonstrate that these topological modes are robust even in the presence strong interactions, leading to a strongly correlated topological many-particle state. Our results put forward quantum dot arrays as a platform for engineering non-Hermitian many-body topological modes, and highlight the resilience of non-Hermitian topology to electronic interactions.
\end{abstract}

DOI: 10.1103/PhysRevResearch.4.L012006

Non-Hermitian (NH) phenomena emerging in artificially designed systems have motivated the rise of a new family of topological states [1,2]. On a mean-field level and in the case of specific types of Lindbladian dynamics, open quantum systems, experiencing gain and loss of energy due to coupling to the environment, can be described with $\mathrm{NH}$ Hamiltonians [1-9]. Such approach has provided profound insights for the description of $\mathrm{NH}$ topological phases, the unconventional NH bulk-boundary correspondence, and the skin effect. Paradigmatic experiments probing the NH topology have concentrated on photonic systems and electrical circuits [10-19]. However, theoretical works have demonstrated that $\mathrm{NH}$ topological phases can be realized also in fermionic systems and superconductors, where the NH self-energy arises due to coupling to a reservoir [20-25].

Here, we study $\mathrm{NH}$ quantum dots $[26,27]$ as a platform to engineer a correlated $\mathrm{NH}$ topological state. In comparison to the other strongly correlated systems the advantage of the quantum dots is the possibility to engineer a modulation of dissipation by coupling the quantum dots to normal metal leads via gate-tunable quantum point contacts (see Fig. 1). These types of tunable quantum dot arrays have been experimentally realized [28-30] and previously considered for adaptive engineering of Hermitian topological phases [31]. We demonstrate that the $\mathrm{NH}$ topological model can be realized in the quantum dot chain and we show that the end modes are resilient to strong electronic interactions by solving the model with a $\mathrm{NH}$ tensor-network algorithm. We show that ultimately the emergence of the end modes is associated with a topological degeneracy of the many-body interacting non-Hermitian

Published by the American Physical Society under the terms of the Creative Commons Attribution 4.0 International license. Further distribution of this work must maintain attribution to the author(s) and the published article's title, journal citation, and DOI. model and we discuss the resulting experimental signatures in the tunneling spectroscopy. Because the gate-tunability of the quantum dots allows us to control the parameters of the lowenergy theory, including the interactions and the dissipation, our results put forward quantum dot arrays as model systems for investigating the interplay of $\mathrm{NH}$ topology and many-body interactions.

The NH model, tackled in the quantum dot platform, is described by an effective $\mathrm{NH}$ Bloch Hamiltonian $\mathcal{H}_{0}=$ $\sum_{k} c_{k, \alpha}^{\dagger} H_{k}^{\alpha, \beta} c_{k, \beta}$, where

$$
H_{k}=\left(\begin{array}{cccc}
i g_{1} & t & 0 & t e^{-i k} \\
t & i g_{2} & t & 0 \\
0 & t & i g_{3} & t \\
t e^{i k} & 0 & t & i g_{4}
\end{array}\right)
$$

$t$ is the nearest-neighbor hopping amplitude and the nontrivial topology arises from the on-site non-Hermiticity $i g_{i}$ [32-34]. While this model supports a family of topological phases [32-34], we focus on the case $g_{1}=g_{4}=0$ and $g_{2}=$ $g_{3}=-2 g$. In this case, the topology is purely induced by dissipation, but we point out that the model is topologically equivalent to a balanced gain-loss model $g_{1}=g_{4}=g$ and $g_{2}=g_{3}=-g$ [see Fig. 1(b)], because the latter is obtained from the previous one by shifting the imaginary parts of the energies by a constant. We use these two models interchangeably but when calculating the observables, which are influenced by the lifetime of the excitations, we use the correct imaginary parts of the on-site energies [35].

The Hamiltonian $H_{k}$ satisfies a $\mathrm{NH}$ chiral symmetry $\mathcal{S} H_{k} \mathcal{S}=-H_{k}^{\dagger}$ with $\mathcal{S}=\operatorname{diag}(1,-1,1,-1)$, and therefore the topology of this $1 \mathrm{D}$ system is described by Chern number $C$ [33] in strong contrast to the Hermitian systems where the Chern number describes the topology of 2D systems [36]. The Chern number is $C=-1$ for the parameters described above giving rise to $\mathrm{NH}$ topological end modes with zero real part of the energy $[33,35]$. These end modes are shown in Figs. 2(a) 
(a)

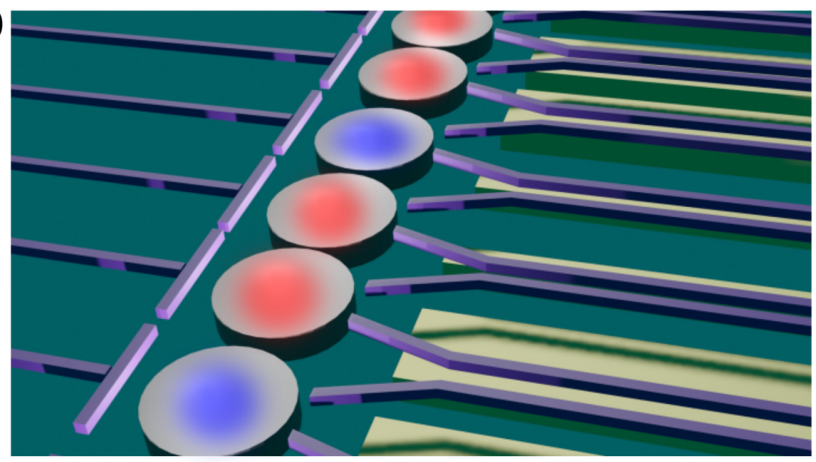

(b)

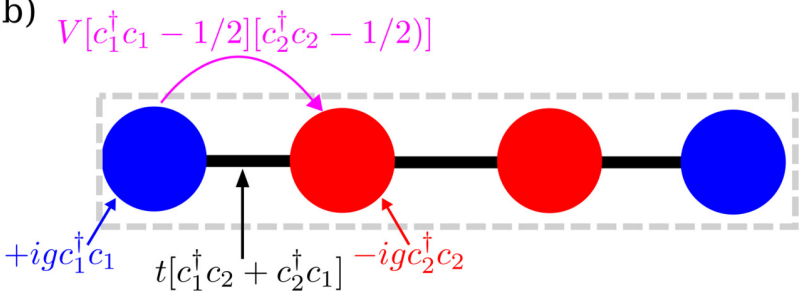

FIG. 1. Schematic illustration of the tunable NH quantum dot chain. The split-gate quantum point contacts control the NH selfenergies $i g_{i}$ via the tunnel couplings between the quantum dots and the normal metal leads. The interaction strengths $V$ can be controlled either with the sizes of the quantum dots or by utilizing the screening of the normal metal leads. Additional gates can be used for tuning the energies of the quantum dot states and the tunneling $t$ between the quantum dots.

and 2(b) together with the bulk bands as a function of $g$. Although $C=-1$ for all values of $g$, increasing the dissipation leads to stronger localization of the zero modes to the end of the chain and, counterintuitively, increases the lifetime of these excitations [35]. For $g \sim t$ the localization length is few lattice sites enabling the study of these topological excitations in short chains. If not stated otherwise, we use $g=t$ in our numerical calculations.

Quantum dot systems provide a natural way of realizing the model described above. Our starting point are the generic quantum dots, which do not possess any symmetries, so that spacing between the discrete energy levels is large and we can consider a single state in each quantum dot. Spin degeneracy is broken by an applied magnetic field, and we assume that tunneling amplitudes and interactions, and hence the topological gap, are smaller than the Zeeman energy scale. In typical semiconducting quantum dots a Zeeman splitting $\sim 1 \mathrm{meV}$ can be induced by applying a magnetic field $B=10 \mathrm{~T}$, so that we estimate that a topological gap $\sim 0.1 \mathrm{meV}$ can be achieved in these systems by tuning the other parameters of the model. This is sufficient for the experimental study of the NH topology, but we point out that much bigger topological gaps are possible in magnetic semiconductors and materials with large $g$ factor. The most important part of the model is, however, the site-dependent non-Hermiticity, which can be achieved by utilizing a gate-tunable self-energy of the quantum dot state $i$ arising due to coupling to the normal metal lead [35,37-39]:

$$
\Sigma_{i}(E)=\lim _{\eta \rightarrow 0} \sum_{k}\left|t_{i, k}\right|^{2} \frac{1}{E+i \eta-\epsilon_{k}} .
$$

Here $\epsilon_{k}$ is the eigenenergy of state $k$ in the lead and $t_{i, k}$ describes the tunneling amplitude from the quantum dot state $i$ to the state $k$ in the lead. The self-energy can be written as $\Sigma_{i}(E)=\tilde{E}_{i}-i g_{i}$, where $\tilde{E}_{i}$ renormalizes the energy of the quantum dot state and

$$
g_{i}(E)=\lim _{\eta \rightarrow 0} \sum_{k}\left|t_{i, k}\right|^{2} \frac{\eta}{\left(E-\epsilon_{k}\right)^{2}+\eta^{2}}
$$

describes the finite lifetime of the electrons in the quantum dot. At low energies, $g_{i}(E)$ is independent of the energy and described by $g_{i}=\pi\left|t_{i N}\right|^{2} \rho$, where $t_{i N}$ is the effective tunneling amplitude and $\rho$ is the density of states in the lead [38]. Thus, the dissipation $g_{i}$ in each quantum dot $i$ is sensitive to the voltage applied to a split-gate quantum point contact which determines the value of $t_{i N}$ (see Fig. 1). The coupling of the quantum dots to the leads creates a fluctuating number of particles within the quantum dot chain, but by applying gate voltages the average density of electrons in the quantum dots can be externally controlled. Finally, we include the effects of electron-electron interactions. Within our low-energy model the dominating interaction term is

$$
\mathcal{H}_{I}=V \sum_{n}\left(c_{n}^{\dagger} c_{n}-1 / 2\right)\left(c_{n+1}^{\dagger} c_{n+1}-1 / 2\right),
$$

where $V$ describes the strength of the electron-electron interaction between the nearest-neighbor quantum dots. The Hamiltonian $\mathcal{H}=\mathcal{H}_{0}+\mathcal{H}_{I}$, consisting of the noninteracting part $\mathcal{H}_{0}$ [Eq. (1)] and interactions $\mathcal{H}_{I}$ [Eq. (4)], is the generic low-energy theory of the $\mathrm{NH}$ quantum dot chain. All parameters of the model are tunable. The split-gate quantum point contacts control the $\mathrm{NH}$ self-energies $i g_{i}$, the interaction strengths $V$ can be controlled either with the sizes of the quantum dots or by utilizing the screening of the normal metal leads, and additional gates can be used for tuning the energies of the quantum dot states and the tunneling $t$ between the quantum dots.

In the case of interacting system we do not have direct access to the single-particle end state wave functions, but instead we have to express the quantities of interest using the manyparticle wave functions. In the noninteracting case, there exist 4 many-particle states which all have the same real part of the energy up to the exponentially small corrections arising due to the finite-size effects, and we can explicitly construct these states by considering the cases where each end mode is either occupied or unoccupied [35]. We find that this 4-fold degeneracy of the real parts of the energies survives in the presence of interactions. Moreover, it resembles the topological degeneracies arising in many-particle Hermitian systems because it distinguishes the system with open boundary conditions from the system with periodic boundary conditions. In addition to the existence of the topological degeneracy we would also like to demonstrate that the many-particle excitations are localized at the end of the chain. For this purpose, we denote the manyparticle state with smallest real part of the energy as $|\Omega\rangle$ and the three other states with almost equal real parts of the energy as $\left|\Psi_{i}\right\rangle(i=-1,0,1)$, and we compute the local correlator defined as

$$
\Xi(n)=\sum_{i}\left|\left\langle\Psi_{i}\left|c_{n}\right| \Omega\right\rangle\right|^{2} .
$$



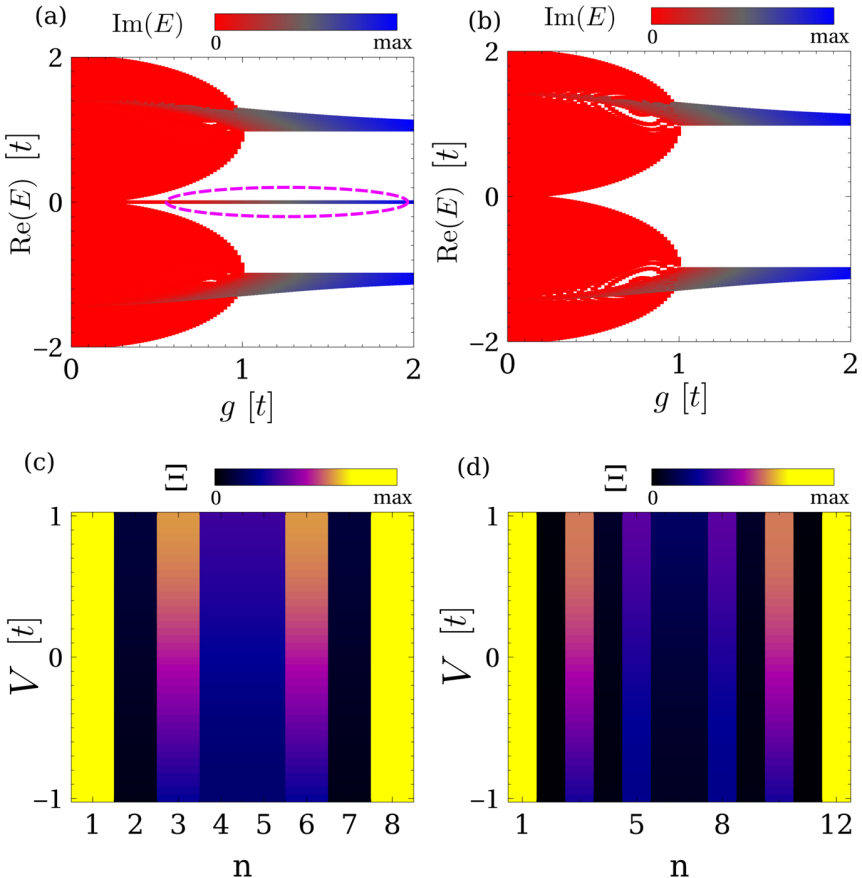

FIG. 2. (a), (b) Spectra for the noninteracting chain with (a) open and (b) periodic boundary conditions. The open chain supports topological end modes with zero real part of the energy. (c), (d) Local correlator $\Xi(n)$, calculated using exact diagonalization of the interacting Hamiltonian, as a function of the interaction strength $V$ demonstrating the resilience of zero modes to interactions. The chain lengths are $L=400$ in (a) and (b), $L=8$ in (c), and $L=12$ in (d).

In noninteracting Hermitian systems, $\Xi(n)$ would yield the wave function of the topological end states and the local zero-bias tunneling conductance would satisfy $d I / d V(\omega=$ $0, x) \sim \Xi(x)$. Due to these reasons, the local correlator $\Xi(n)$ is expected to yield information about the localization of the many-particle excitations. This indeed turns out to be the case, but it is worth pointing out that the analogies to the Hermitian systems are not perfect. First, the eigenstates of the noninteracting NH Hamiltonian are not orthogonal to each other, and therefore the local correlator $\Xi(n)$ does not exactly describe the end state wave functions even in the noninteracting limit. Nevertheless, $\Xi(n)$ serves as a good indicator of the localization properties of the excitations [35]. Second, the excitations in NH systems have finite lifetimes, which show up as broadening of the peaks in the differential conductance. Therefore, the zero-bias differential conductance also includes a contribution from bulk states. Nevertheless, the bulk states are delocalized in the whole sample and therefore end excitations show up as a broadened zero-bias peak in the differential conductance in the vicinity of the end of the chain [35]. Therefore, $\Xi(n)$ as a function of $n$ serves as a good proxy for the local tunneling conductance profile.

Figures 2(c) and 2(d) show the local correlator $\Xi(n)$, obtained from exact diagonalization calculations, as a function of the interaction strength $V$ for short chains. The local correlator shows the emergence of topological end modes and demonstrates their resilience even in the presence of very strong interactions $V=t$, which leads to a full restructuring (a)

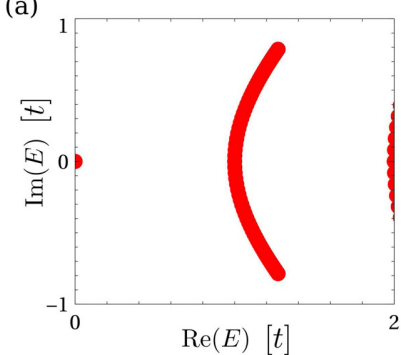

(b)

(c)
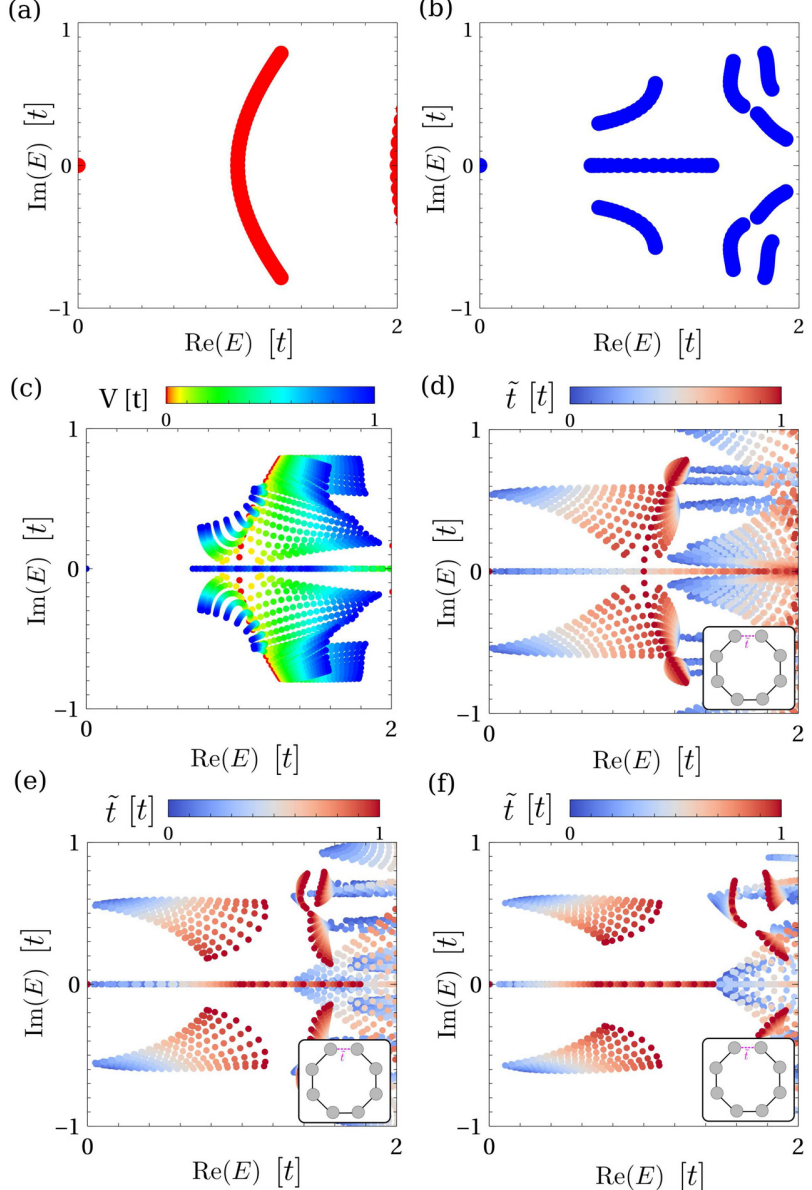

(f)

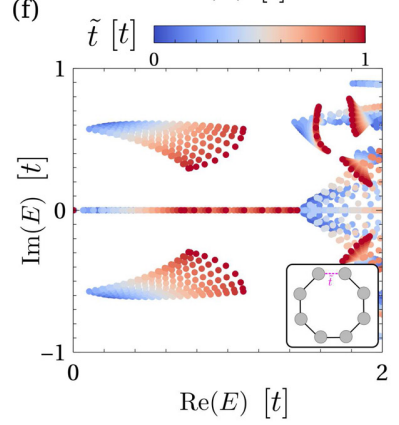

FIG. 3. (a), (b) Many-body energies relative to the lowest real part of the energy (averaged over twisted boundary conditions) for (a) $V=0$ and (b) $V=t$ with closed boundary conditions. (c) The evolution of the energies as $V$ is ramped up. (d)-(f) The evolution of the energies as the boundary conditions are changed from closed $(\tilde{t}=t)$ to open $(\tilde{t}=0)$ for (d) $V=0$, (e) $V=0.5 t$, and (f) $V=t$. We used $L=8$, but to simulate the energy bands we collected the energies corresponding to the different twisted boundary conditions.

of the bulk bands as shown in Figs. 3(a) and 3(b), where the many-body energies are measured relative to the lowest real part of the energy (averaged over twisted boundary conditions). To understand the robustness of the topological end modes, we notice that the bulk gap (line gap with respect to real part of the energies [6]) remains open upon increasing interactions from $V=0$ to $V=t$, demonstrating the existence an adiabatic connection between the noninteracting and interacting limits [Fig. 3(c)]. We have further elaborated the correspondence between the end modes in the noninteracting and strongly interacting limits by demonstrating that the evolution of the spectrum is qualitatively similar for all values of $V$ when one of the hopping amplitudes is varied from closed $(\tilde{t}=t)$ to open $(\tilde{t}=0)$ boundary conditions [Figs. 3(d)-3(f)]. In the case of open boundary conditions, we always observe the emergence of the 4-fold many-body degeneracy in the real part of the energies, and therefore we interpret this degeneracy as the many-body signature of the topological phase. The 


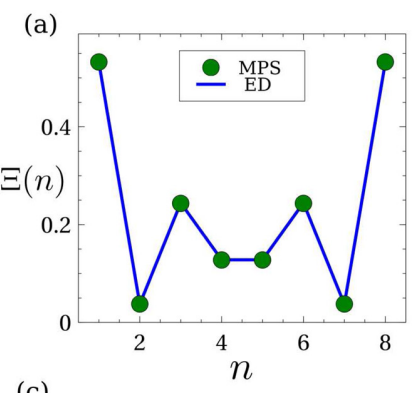

(b)

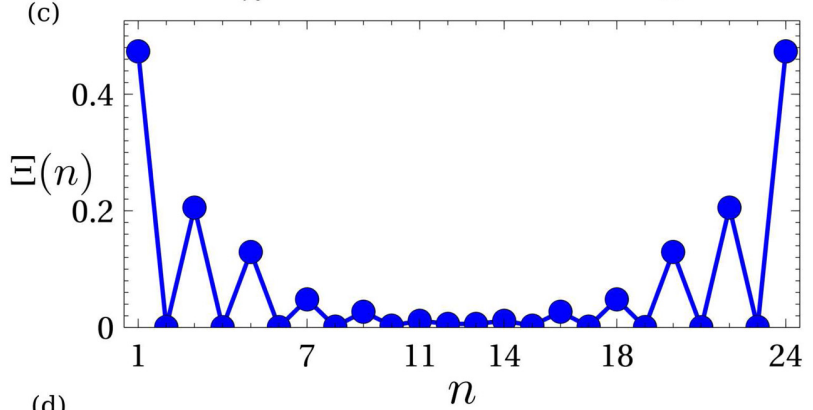

(d)

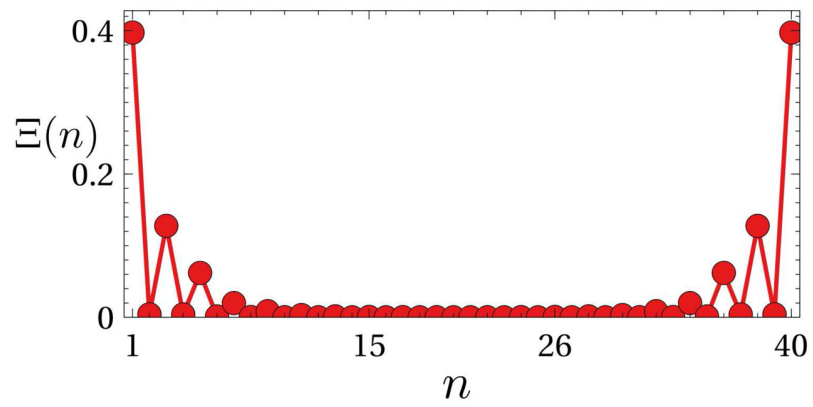

FIG. 4. (a), (b) Comparison between $\Xi(n)$ obtained using exact diagonalization and the tensor-network formalism for short chains (a) $L=8$ and (b) $L=12$. (c), (d) $\Xi(n)$ calculated using the tensornetwork formalism for long chains (c) $L=24$ and (d) $L=40$. The local correlator vanishes in the bulk implying that the low-energy excitations are localized at the end of the chain also in a strongly interacting system. We used $V=t$.

imaginary parts of the many-body energies are different due to the finite lifetime of the end modes.

To get rid of the finite-size effects, we have studied longer chains using a tensor-network formalism [40-42]. In our calculations, the NH many-body eigenstates were represented as tensor networks, and we implemented a NH Krylov subspace diagonalization [43-47] targeting the many-body states with the lowest real parts of the energies. It is worth noting that, while Hermitian many-body Hamiltonians are commonly solved with tensor-network techniques using density-matrix renormalization algorithms, those algorithms are not directly applicable in the non-Hermitian limit. In contrast, a non-Hermitian Arnoldi algorithm within the tensor-network formalism allows solving interacting non-Hermitian problems. To benchmark the algorithm, we show in Figs. 4(a) and 4(b) that for a short chain the local correlator obtained with exact diagonalization and tensor-network formalism show a perfect agreement. In particular, we observe the existence of end modes in the spatial profile of $\Xi(n)$. The important advantage of the tensor-network formalism is that we can solve the many-body eigenstates for much longer chains. The results for $L=20$ and $L=40$ are shown in Figs. 4(c) and 4(d). They demonstrate that $\Xi(n)$ vanishes in the bulk, implying that the low-energy excitations are localized at the end of the chain also in a strongly interacting system with $V=t$.

To summarize, we have proposed quantum dot chains as a viable experimental platform for studying interacting $\mathrm{NH}$ topological phases, and by performing calculations using exact diagonalization and tensor-network algorithms, we have demonstrated that the topological end modes exists in the presence of strong interactions and lead to a robust 4-fold topological degeneracy of the real parts of the many-particle energies. Our results put forward a paradigmatic example of interacting topological matter, opening up interesting directions in the many-body $\mathrm{NH}$ physics. In particular, we have demonstrated this phenomenology in a minimal interacting $\mathrm{NH}$ model, whose topological end modes arise from a nontrivial Chern number $|C|=1$. More generally, the Chern number is an integer topological invariant, and it is possible to construct models for free-fermion NH systems with arbitrarily large Chern numbers [35]. The topological classification of the interacting $\mathrm{NH}$ systems goes beyond the scope of this work, but we point out that the fate of the higher Chern number topological phases in interacting $\mathrm{NH}$ systems is a genuinely interesting problem for future research. In general, interactions can destroy the protection of the topological end modes because spontaneous symmetry breaking can make the topological invariant ill defined or result in an appearance of a skin effect. On the other hand, it is also known that in Hermitian systems the interactions can lead to a collapse of the classification of the free-fermion systems due to effects going beyond the mean-field theory [48-50]. If we assume that there is no spontaneous symmetry breaking and there is no collapse of the classification of the free-fermion systems (as indicated by our calculations in the case of $|C|=1$ and the range of interaction strengths considered in our work), then the topological end modes are protected if there exists an adiabatic continuity between the noninteracting and strongly interacting limits as the interactions are ramped up (as found in our calculations). In interacting Hermitian systems, the many-body Chern number can be computed in various ways including the Green's function formalism [51-57], and assuming that the Green's function at zero frequency satisfies the $\mathrm{NH}$ chiral symmetry this formalism generalizes to the family of interacting $\mathrm{NH}$ systems studied in our Letter. Therefore, assuming that similar adiabatic continuity persists also in the case of higher Chern numbers, the topological end modes in the higher Chern number topological phases may also be resilient to strong interactions.

We acknowledge the computational resources provided by the Aalto Science-IT project. J.L.L. acknowledges financial support from the Academy of Finland Projects No. 331342 and No. 336243. The research was also partially supported by the Foundation for Polish Science through the IRA Programme co-financed by EU within SG OP. 
[1] Y. Ashida, Z. Gong, and M. Ueda, Non-Hermitian physics, Adv. Phys. 69, 249 (2020).

[2] E. J. Bergholtz, J. C. Budich, and F. K. Kunst, Exceptional topology of non-Hermitian systems, Rev. Mod. Phys. 93, 015005 (2021).

[3] R. El-Ganainy, K. G. Makris, M. Khajavikhan, Z. H. Musslimani, S. Rotter, and D. N. Christodoulides, NonHermitian physics and PT symmetry, Nat. Phys. 14, 11 (2018).

[4] F. Song, S. Yao, and Z. Wang, Non-Hermitian Skin Effect and Chiral Damping in Open Quantum Systems, Phys. Rev. Lett. 123, 170401 (2019).

[5] S. Lieu, M. McGinley, and N. R. Cooper, Tenfold Way for Quadratic Lindbladians, Phys. Rev. Lett. 124, 040401 (2020).

[6] K. Kawabata, K. Shiozaki, M. Ueda, and M. Sato, Symmetry and Topology in Non-Hermitian Physics, Phys. Rev. X 9, 041015 (2019).

[7] P. M. Vecsei, M. M. Denner, T. Neupert, and F. Schindler, Symmetry indicators for inversion-symmetric non-Hermitian topological band structures, Phys. Rev. B 103, L201114 (2021).

[8] K. Sponselee, L. Freystatzky, B. Abeln, M. Diem, B. Hundt, A. Kochanke, T. Ponath, B. Santra, L. Mathey, K. Sengstock, and C. Becker, Dynamics of ultracold quantum gases in the dissipative Fermi-Hubbard model, Quantum Sci. Technol. 4, 014002 (2018).

[9] S. H. Cantu, A. V. Venkatramani, W. Xu, L. Zhou, B. Jelenković, M. D. Lukin, and V. Vuletić, Repulsive photons in a quantum nonlinear medium, Nat. Phys. 16, 921 (2020).

[10] T. Ozawa, H. M. Price, A. Amo, N. Goldman, M. Hafezi, L. Lu, M. C. Rechtsman, D. Schuster, J. Simon, O. Zilberberg, and I. Carusotto, Topological photonics, Rev. Mod. Phys. 91, 015006 (2019).

[11] J. M. Zeuner, M. C. Rechtsman, Y. Plotnik, Y. Lumer, S. Nolte, M. S. Rudner, M. Segev, and A. Szameit, Observation of a Topological Transition in the Bulk of a Non-Hermitian System, Phys. Rev. Lett. 115, 040402 (2015).

[12] C. Poli, M. Bellec, U. Kuhl, F. Mortessagne, and H. Schomerus, Selective enhancement of topologically induced interface states in a dielectric resonator chain, Nat. Commun. 6, 6710 (2015).

[13] X. Zhan, L. Xiao, Z. Bian, K. Wang, X. Qiu, B. C. Sanders, W. Yi, and P. Xue, Detecting Topological Invariants in Nonunitary Discrete-Time Quantum Walks, Phys. Rev. Lett. 119, 130501 (2017).

[14] L. Xiao, X. Zhan, Z. H. Bian, K. K. Wang, X. Zhang, X. P. Wang, J. Li, K. Mochizuki, D. Kim, N. Kawakami, W. Yi, H. Obuse, B. C. Sanders, and P. Xue, Observation of topological edge states in parity-time-symmetric quantum walks, Nat. Phys. 13, 1117 (2017).

[15] S. Weimann, M. Kremer, Y. Plotnik, Y. Lumer, S. Nolte, K. G. Makris, M. Segev, M. Rechtsman, and A. Szameit, Topologically protected bound states in photonic parity-time-symmetric crystals, Nat. Mater. 16, 433 (2017).

[16] H. Zhao, P. Miao, M. H. Teimourpour, S. Malzard, R. ElGanainy, H. Schomerus, and L. Feng, Topological hybrid silicon microlasers, Nat. Commun. 9, 981 (2018).

[17] M. A. Bandres, S. Wittek, G. Harari, M. Parto, J. Ren, M. Segev, D. N. Christodoulides, and M. Khajavikhan, Topological insulator laser: Experiments, Science 359, eaar4005 (2018).

[18] M. Parto, S. Wittek, H. Hodaei, G. Harari, M. A. Bandres, J. Ren, M. C. Rechtsman, M. Segev, D. N. Christodoulides, and
M. Khajavikhan, Edge-Mode Lasing in 1D Topological Active Arrays, Phys. Rev. Lett. 120, 113901 (2018).

[19] T. Helbig, T. Hofmann, S. Imhof, M. Abdelghany, T. Kiessling, L. W. Molenkamp, C. H. Lee, A. Szameit, M. Greiter, and R. Thomale, Generalized bulk-boundary correspondence in nonHermitian topolectrical circuits, Nat. Phys. 16, 747 (2020).

[20] D. I. Pikulin and Y. V. Nazarov, Topological properties of superconducting junctions, JETP Lett. 94, 693 (2012).

[21] D. I. Pikulin and Y. V. Nazarov, Two types of topological transitions in finite Majorana wires, Phys. Rev. B 87, 235421 (2013).

[22] S. Mi, D. I. Pikulin, M. Marciani, and C. W. J. Beenakker, $\mathrm{X}$-shaped and Y-shaped Andreev resonance profiles in a superconducting quantum dot, J. Exp. Theor. Phys. 119, 1018 (2014).

[23] P. San-Jose, J. Cayao, E. Prada, and R. Aguado, Majorana bound states from exceptional points in non-topological superconductors, Sci. Rep. 6, 21427 (2016).

[24] J. Avila, F. Peñaranda, E. Prada, P. San-Jose, and R. Aguado, Non-hermitian topology as a unifying framework for the Andreev versus Majorana states controversy, Commun. Phys. 2 133 (2019).

[25] E. J. Bergholtz and J. C. Budich, Non-Hermitian Weyl physics in topological insulator ferromagnet junctions, Phys. Rev. Research 1, 012003(R) (2019).

[26] D. Ferry, R. Akis, A. Burke, I. Knezevic, R. Brunner, J. Bird, R. Meisels, F. Kuchar, and J. Bird, Open quantum dots: Physics of the non-Hermitian Hamiltonian, Fortschr. Phys. 61, 291 (2012).

[27] L.-L. Zhang, G.-H. Zhan, D.-Q. Yu, and W.-J. Gong, Transport through a non-Hermitian parallel double-quantum-dot structure in the presence of interdot Coulomb interaction, Superlattices Microstruct. 113, 558 (2018).

[28] T. Hensgens, T. Fujita, L. Janssen, X. Li, C. J. Van Diepen, C. Reichl, W. Wegscheider, S. Das Sarma, and L. M. K. Vandersypen, Quantum simulation of a Fermi-Hubbard model using a semiconductor quantum dot array, Nature (London) 548, 70 (2017).

[29] A. R. Mills, D. M. Zajac, M. J. Gullans, F. J. Schupp, T. M Hazard, and J. R. Petta, Shuttling a single charge across a onedimensional array of silicon quantum dots, Nat. Commun. 10, 1063 (2019).

[30] P.-A. Mortemousque, B. Jadot, E. Chanrion, V. Thiney, C. Bäuerle, A. Ludwig, A. D. Wieck, M. Urdampilleta, and T. Meunier, Enhanced spin coherence while displacing electron in a 2D array of quantum dots, PRX Quantum 2, 030331 (2021).

[31] I. C. Fulga, A. Haim, A. R. Akhmerov, and Y. Oreg, Adaptive tuning of Majorana fermions in a quantum dot chain, New J. Phys. 15, 045020 (2013).

[32] K. Takata and M. Notomi, Photonic Topological Insulating Phase Induced Solely by Gain and Loss, Phys. Rev. Lett. 121 213902 (2018).

[33] W. Brzezicki and T. Hyart, Hidden Chern number in onedimensional non-Hermitian chiral-symmetric systems, Phys. Rev. B 100, 161105(R) (2019).

[34] P. Comaron, V. Shahnazaryan, W. Brzezicki, T. Hyart, and M. Matuszewski, Non-Hermitian topological end-mode lasing in polariton systems, Phys. Rev. Research 2, 022051(R) (2020).

[35] See Supplemental Material at http://link.aps.org/supplemental/ 10.1103/PhysRevResearch.4.L012006 for more details related 
to analytical results in the non-interacting limit, topological invariant, derivation of the non- Hermitian self-energy, end state wave functions and local correlator in the absence of interactions, tunneling conductance, models for topological phases with arbitrary large Chern number, and robustness of the topological end modes in the presence of interactions.

[36] D. J. Thouless, M. Kohmoto, M. P. Nightingale, and M. den Nijs, Quantized Hall Conductance in a Two-Dimensional Periodic Potential, Phys. Rev. Lett. 49, 405 (1982).

[37] C. W. J. Beenakker, Random-matrix theory of quantum transport, Rev. Mod. Phys. 69, 731 (1997).

[38] S. Datta, Quantum Transport: Atom to Transistor (Cambridge University Press, Cambridge, 2013).

[39] H. Bruus and K. Flensberg, Many-Body Quantum Theory in Condensed Matter Physics: An Introduction, Oxford Graduate Texts (Oxford University Press, Oxford, 2016).

[40] M. Fishman, S. R. White, and E. M. Stoudenmire, The ITensor software library for tensor network calculations, arXiv:2007.14822.

[41] ITensor Library, http://itensor.org.

[42] DMRGpy Library, https://github.com/joselado/dmrgpy.

[43] W. E. Arnoldi, The principle of minimized iterations in the solution of the matrix eigenvalue problem, Q. Appl. Math. 9, 17 (1951).

[44] R. B. Lehoucq and D. C. Sorensen, Deflation techniques for an implicitly restarted Arnoldi iteration, SIAM J. Matrix Anal. Appl. 17, 789 (1996).

[45] F. Tisseur and K. Meerbergen, The quadratic eigenvalue problem, SIAM Rev. 43, 235 (2001).

[46] A. Stathopoulos, Y. Saad, and K. Wu, Dynamic thick restarting of the Davidson, and the implicitly restarted Arnoldi methods, SIAM J. Sci. Comput. 19, 227 (1998).
[47] R. B. Lehoucq, D. C. Sorensen, and C. Yang, ARPACK Users' Guide: Solution of Large-Scale Eigenvalue Problems with Implicitly Restarted Arnoldi Methods (SIAM, Philadelphia, 1998).

[48] L. Fidkowski and A. Kitaev, Effects of interactions on the topological classification of free fermion systems, Phys. Rev. B 81, 134509 (2010).

[49] L. Fidkowski and A. Kitaev, Topological phases of fermions in one dimension, Phys. Rev. B 83, 075103 (2011).

[50] C.-K. Chiu, J. C. Y. Teo, A. P. Schnyder, and S. Ryu, Classification of topological quantum matter with symmetries, Rev. Mod. Phys. 88, 035005 (2016).

[51] G. E. Volovik, Topological invariant for superfluid $3 \mathrm{He}-\mathrm{B}$ and quantum phase transitions, JETP Lett. 90, 587 (2009).

[52] V. Gurarie, Single-particle Green's functions and interacting topological insulators, Phys. Rev. B 83, 085426 (2011).

[53] Z. Wang and S.-C. Zhang, Simplified Topological Invariants for Interacting Insulators, Phys. Rev. X 2, 031008 (2012).

[54] Z. Wang and B. Yan, Topological Hamiltonian as an exact tool for topological invariants, J. Phys.: Condens. Matter 25, 155601 (2013).

[55] T. Mertz, K. Zantout, and R. Valentí, Statistical analysis of the Chern number in the interacting Haldane-Hubbard model, Phys. Rev. B 100, 125111 (2019).

[56] D. Lessnich, S. M. Winter, M. Iraola, M. G. Vergniory, and R. Valentí, Elementary band representations for the single-particle Green's function of interacting topological insulators, Phys. Rev. B 104, 085116 (2021).

[57] Y.-Z. You, Z. Wang, J. Oon, and C. Xu, Topological number and fermion Green's function for strongly interacting topological superconductors, Phys. Rev. B 90, 060502(R) (2014). 